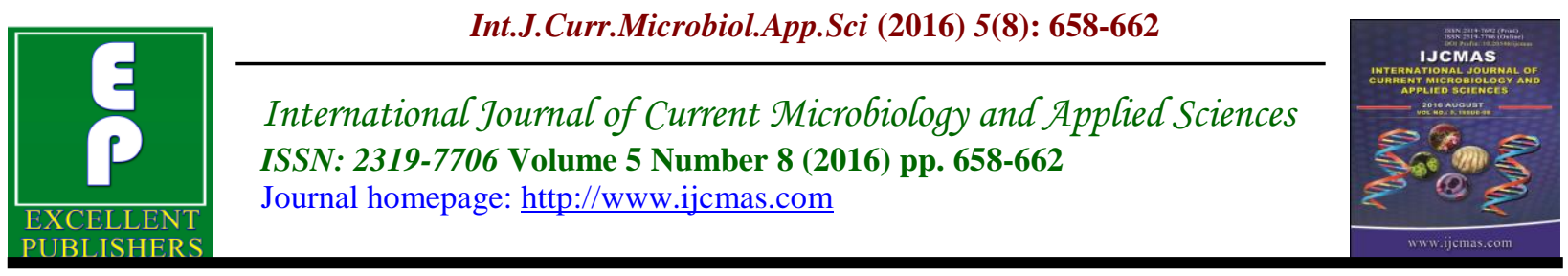

Original Research Article

http://dx.doi.org/10.20546/ijcmas.2016.508.074

\title{
Isolation and Antibiogram of Enterococci from Patients with Urinary Tract Infection in a Tertiary Care Hospital
}

\author{
Siva Prasad Reddy Basava* \\ Department of Microbiology, Narayana Medical College, Nellore, Andhra Pradesh, India \\ *Corresponding author
}

\begin{abstract}
A B S T R A C T
Keywords

Enterococci, UTI, Linezolid, Vancomycin

Resistant

Enterococci,

Tigecycline,

Teicoplanin.

Article Info

Accepted:

25 July 2016

Available Online:

10 August 2016

Enterococci are implicated in blood stream infections (BSI), endocarditis, urinary tract infections (UTI), pyogenic infections, intra-abdominal and pelvic infections. The most common nosocomial infections produced by these organisms are urinary tract infections (associated with instrumentation and antimicrobial administration), followed by intra-abdominal and pelvic infections. The present study was conducted to analyse the changing patterns of antibiotic sensitivity to Enterococci in patients with urinary tract infection. It was a prospective study of one year duration where 1180 urine samples from patients with suspected UTI were analysed. Isolation of Enterococci was done by conventional methods and AST was done by Kirby Bauer Disc Diffusion method as per CLSI guidelines. Linezolid would be the best choice for treatment of complicated and multidrug resistant enterococcal infections. For vancomycin resistant Enterococci, tigecycline can be a good option. Teicoplanin can be used as a reserve drug for enterococcal infections. Azithromycin and Clindamycin have no therapeutic role in treatment of enterococcal infections and hence can be avoided while antibiotic susceptibility testing.
\end{abstract}

\section{Introduction}

The genus Enterococcus consists of Grampositive, facultatively anaerobic organisms that are ovoid in shape and may appear on smear in short chains, in pairs or as single cells. It forms an indigenous flora of the intestinal tract, oral cavity and the genitourinary tract of the humans and animals, are known to be relatively avirulent in healthy individuals, but have become important opportunistic pathogens, especially in hospitalized patients. Enterococci are implicated in blood stream infections (BSI), endocarditis, urinary tract infections (UTI), pyogenic infections, intraabdominal and pelvic infections (Murray et al., 1990). The most common nosocomial infections produced by these organisms are urinary tract infections (associated with instrumentation and antimicrobial administration), followed by intraabdominal and pelvic infections (Marothi et al., 2005). A common regime for treatment of serious enterococcal infections is the combination of cell-wall inhibitors, such as 
penicillin, ampicillin or vancomycin; with aminoglycosides, such as streptomycin or gentamicin. The addition of cell-wall inhibitor agent helps in the penetration of the aminoglycoside into the bacterial cytoplasm, making the intrinsically resistant organism aminoglycoside sensitive. Reduced susceptibility to vancomycin will interfere with the penetration of the aminoglycoside into the bacterial cytoplasm, thus making the synergism in effective (Herman et al., 1991).It is imperative to analyse the pattern of susceptibility to newer drugs which may be effective to treat Vancomycin Resistant Enterococci. There is a huge dearth of information regarding the effect of Tigecycline and Teicoplanin on Enterococci. This study was undertaken to provide accurate antimicrobial resistance patterns for enterococci so that effective therapy can be initiated from cases of urinary tract infection.

\section{Materials and Methods}

\section{Study population, design and setting}

The present study was a prospective cross sectional study conducted in the department of Microbiology at Narayana Medical College, Nellore over a 1 year period from July 2015 to June 2016.

\section{Inclusion Criteria}

1) Patients with symptoms of UTI

2) Patients from both Outpatient and Inpatient wards

\section{Exclusion Criteria}

1) Patients on antibiotic therapy

2) Infants

3) Pregnant women

\section{Sample collection}

Mid-stream urine sample in early morning was collected in wide mouth sterile container. Male patients were instructed to cleanse the glans penis with soap and water, dry the area, and collect the urine with foreskin retracted. Female patients were instructed to cleanse the area around the urethral opening with soap and water, dry the area, and collect the urine with the labia held apart.

\section{Identification}

Urine samples were cultured over routine culture media; MacConkey agar and Cysteine lactose electrolyte deficient (CLED) agar with a sterile standard loop. These plates were incubated at $37^{\circ} \mathrm{C}$ for 2 consecutive days. Enterococci were identified on the basis of appearance on gram stain, growth in $6.5 \% \mathrm{NaCI}$, catalasenegative, growth on bile esculin medium. Bacitracin resistance and positive Voges-Proskauer test were also used for the confirmation of isolates as enterococci.

\section{Antibiotic Susceptibility Testing}

Antimicrobial susceptibility testing and interpretation was carried out on MuellerHinton agar (HiMedia Laboratories, India) by standard disc diffusion method as per Clinical Laboratory Standards Institute (CLSI) guidelines using discs of standard concentration. Standard strains of Staphylococcus aureus ATCC 25923 and E. faecalis ATCC 29212 were used as controls.

The antibiotics tested were (concentration in $\mu \mathrm{g})$ as follows: Ampicillin (10), Cefixime (5), Azithromycin (15), Ofloxacin(5), Gentamycin (30), Clindamycin (10), Amoxyclav (30), Piperacillin/Tazobactam 
(100/10), Teicoplanin (30) Tigecycline (15), Vancomycin (30), and Linezolid (30). These discs were obtained from HiMedia laboratories, India.

\section{Results and Discussion}

A total of 1180 urine samples were studied from patients with suspected signs and symptoms of urinary tract infection, out of which 800 specimens were positive in culture. Enterococci were isolated in pure cultures in 115 specimens. Speciation was not done. Enterococci were resistant to Azithromycin (88.9\%) and Clindamycin $(85.7 \%)$, sensitive to Ofloxacin $(95 \%)$, Tigecycline (92\%), Vancomycin (78\%), Amoxyclav (84\%), Piperacillin-Tazobactam (92\%), Teicoplanin(86\%) and Linezolid (96\%). However the sensitivity to Gentamicin was $71.4 \%$ and Cefixime was 55\% and Ampicillin was 55\%.

Fig.1

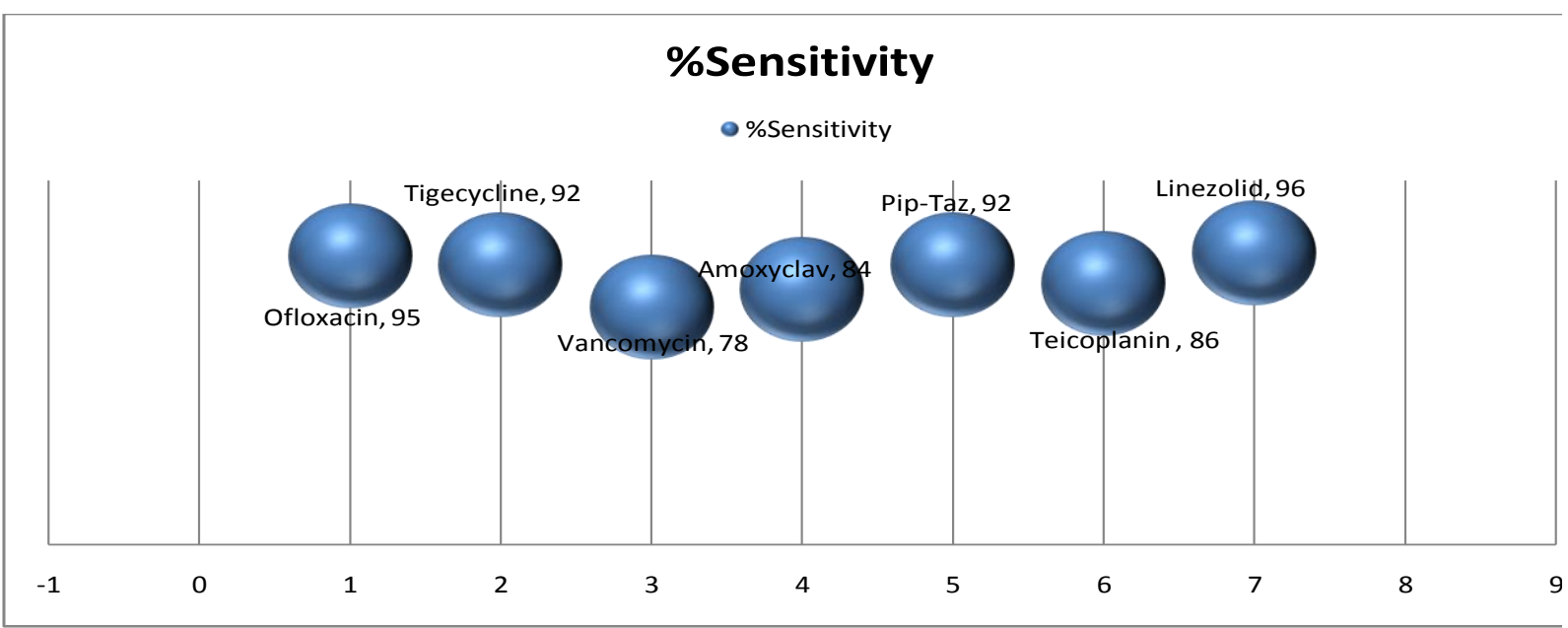

FIG.1 ANTIMICROBIALS SHOWING SENSITIVITY PATTERN

Fig.2

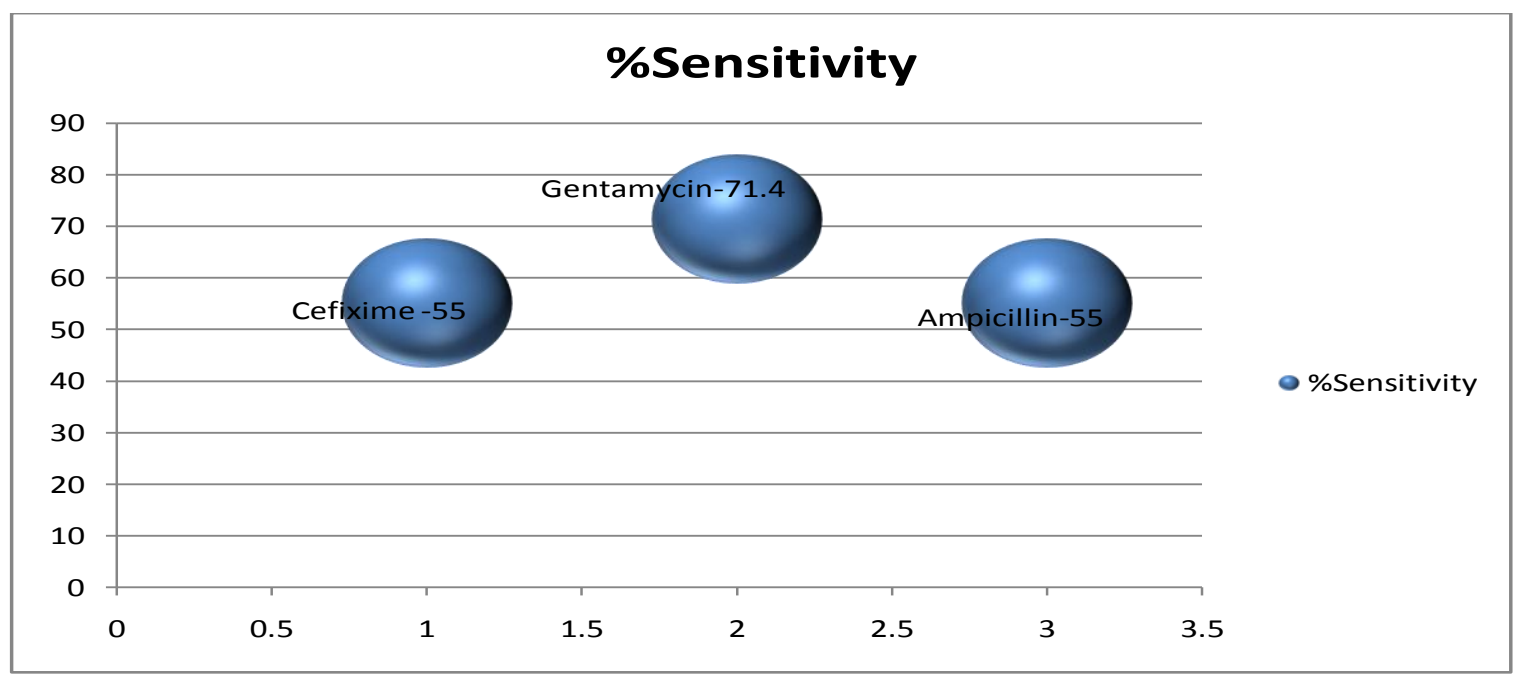

Fig 2 Antimicrobials showing intermediate sensitivity 
Fig.3

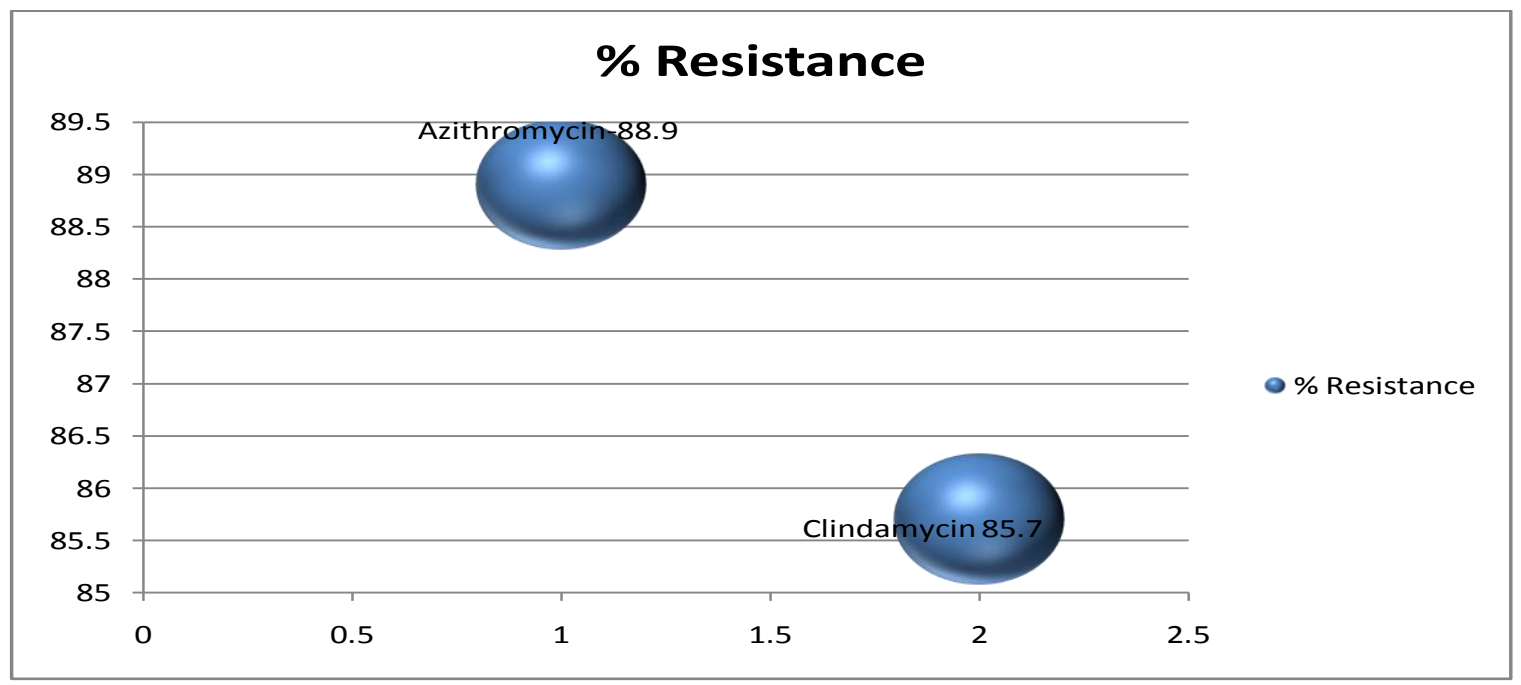

FIG 3. ANTIMICROBIALS SHOWING RESISTANCE PATTERN

The ability to cause serious infections and the pattern of variable resistance to antibiotics has caused increased interest in enterococci in the recent past. In our study the sensitivity to Ampicillin was only 55\% which is in correlation with Jain, et al., (2011) and Tuhina, et al., (2016). Linezolid would be the best drug and the susceptibility patterns were in correlation with Parameswarappa et al., (2013).

We also found that Tigecycline susceptibility is far better than that of Vancomycin making it an alternative for VRE. Vancomycin susceptibility in our study correlated with the study of Gupta P $e t$ al., (2015). However the degree of sensitivity to Tigecycline is less compared to that of Vidyalakshmi et al., (2012) and Wattal et al., (2010) where there is $100 \%$ sensitivity. This may be due to selection pressure as the drug is commonly used for complicated intra abdominal and skin infections in our hospital. Enterococci are susceptible to Ofloxacin in our study compared to Alam et al., (2011). Enterococci are susceptible to teicoplanin. Azithromycin and Clindamycin are ineffective to treat enterococcal infections. There is no emergence of aminoglycoside resistance in my area.

It is therapeutically challenging to treat enterococcal infections due to intrinsic and acquired resistance to vancomycin and production of $\beta$-lactamases. This creates a clamour to implement appropriate infection control measures to decrease the transmission of these microorganisms in hospital settings. Rational and restricted usage of antibiotics decrease the burden of selection pressure and an appropriate antibiogram helps in adequate empirical treatment options. Hence more studies should be conducted to know the diverse mechanisms of antimicrobial resistance patterns in enterococci across the globe.

\section{References}

Alam, M.S., Pillai, P.K., Kapur, P., Pillai, K.K. 2011. Resistant patterns of bacteria isolated from bloodstream infections at a university hospital in Delhi. J. Pharm. Bioall. Sci., 3: 52530. 
Gupta, P., Mandal, J., Krishnamurthy, S., Barathi, D. 2015. Pandit N.Profile of urinary tract infections in paediatric patients. Indian J. Med. Res., 141: 473-7

Herman, D.J., Gerding, D.N. 1991. Screening and treatment of infection caused by resistant Enterococci. Antimicrob. Agents Chemother., 35: 215-9.

Jain, S., Kumar, A., Kashyap, B., Kaur, I.R. 2011. Clinico-epidemiological profile and high-level aminoglycoside resistance in enterococcal septicemia from a tertiary care hospital in east Delhi. Int. J. App. Basic Med. Res., 1: 80-3.

Marothi, Y.A., Agnihotri, H., Dubey, D. 2005. Enterococcal resistance - An overview. Indian J. Med. Microbiol., 23: 214-9.

Murray, B.E. 1990. The life and times of the Enterococcus. Clin. Microbiol. Rev., 3: 46-65.

Parameswarappa, J., Basavaraj, V.P.,
Basavaraj, C.M. 2013. Isolation, identification and antibiogram of enterococci isolated from patients with urinary tract infection. Ann. Afr. Med., 12: $176-817$.

Tuhina, B., Anupurba, S., Karuna, T. 2016. Emergence of antimicrobial resistance and virulence factors among the unusual species of enterococci, from North India. Indian J. Pathol. Microbiol., 59: 50-5.

Vidyalakshmi, P.R., Gopalakrishnan, R., Ramasubramanian, V., Ghafur, K.A., Nambi, P.S., Thirunarayana, M.A. 2012. Clinical, epidemiological and microbiological profile of patients with vancomycin resistant Enterococci from a Tertiary Care Hospital. $J$. Global Infect. Dis., 4: 137-8.

Wattal, C., Goel, N., Oberoi, J.K., Raveendran, R,. Datta, S., Prasad, K.J. 2010. Surveillance of multidrug resistant organisms in a tertiary care hospital in Delhi, India. J. Assoc. Physicians India, 58 Suppl: 32-6.

\section{How to cite this article:}

Siva Prasad Reddy Basava. 2016. Isolation and Antibiogram of Enterococci from Patients with Urinary Tract Infection in a Tertiary Care Hospital. Int.J.Curr.Microbiol.App.Sci. 5(8): 658662. doi: http://dx.doi.org/10.20546/ijcmas.2016.508.074 